\title{
Conception Method
}

National Cancer Institute

\section{Source}

National Cancer Institute. Conception Method. NCI Thesaurus. Code C92930.

The process of conceiving a baby. 